\title{
The influence of 4-thiouridine labeling on pre-mRNA splicing outcomes
}

\author{
Jessie A. C. Altieri, Klemens J. Hertel ${ }^{*}$ * \\ Department of Microbiology and Molecular Genetics, University of California Irvine, Irvine, California, United \\ States of America \\ * khertel@uci.edu
}

\section{Abstract}

Metabolic labeling is a widely used tool to investigate different aspects of pre-mRNA splicing and RNA turnover. The labeling technology takes advantage of native cellular machineries where a nucleotide analog is readily taken up and incorporated into nascent RNA. One such analog is 4-thiouridine (4sU). Previous studies demonstrated that the uptake of $4 \mathrm{sU}$ at elevated concentrations $(>50 \mu \mathrm{M})$ and extended exposure led to inhibition of rRNA synthesis and processing, presumably induced by changes in RNA secondary structure. Thus, it is possible that $4 \mathrm{sU}$ incorporation may also interfere with splicing efficiency. To test this hypothesis, we carried out splicing analyses of pre-mRNA substrates with varying levels of $4 s \mathrm{U}$ incorporation (0-100\%). We demonstrate that increased incorporation of $4 \mathrm{sU}$ into premRNAs decreased splicing efficiency. The overall impact of $4 \mathrm{sU}$ labeling on pre-mRNA splicing efficiency negatively correlates with the strength of splice site signals such as the 3' and the 5' splice sites. Introns with weaker splice sites are more affected by the presence of $4 \mathrm{sU}$. We also show that transcription by T7 polymerase and pre-mRNA degradation kinetics were impacted at the highest levels of $4 \mathrm{sU}$ incorporation. Increased incorporation of $4 \mathrm{sU}$ caused elevated levels of abortive transcripts, and fully labeled pre-mRNA is more stable than its uridine-only counterpart. Cell culture experiments show that a small number of alternative splicing events were modestly, but statistically significantly influenced by metabolic labeling with $4 \mathrm{sU}$ at concentrations considered to be tolerable $(40 \mu \mathrm{M})$. We conclude that at high 4sU incorporation rates small, but noticeable changes in pre-mRNA splicing can be detected when splice sites deviate from consensus. Given these potential 4sU artifacts, we suggest that appropriate controls for metabolic labeling experiments need to be included in future labeling experiments.

Copyright: @ 2021 Altieri, Hertel. This is an open access article distributed under the terms of the Creative Commons Attribution License, which permits unrestricted use, distribution, and reproduction in any medium, provided the original author and source are credited.

Data Availability Statement: All relevant data are within the paper and its S1 Dataset, S1 Raw images files.

Funding: This work was supported by a grant from the Foundation for the National Institutes of Health, awarded to KJH (R01 GM062287). The funders

\section{Introduction}

4-thiouridine (4sU), a uridine analog, is a commonly used metabolic label that can be employed to investigate a wide variety of topics that range from pre-mRNA generation to mRNA degradation. When added to cell medium, $4 \mathrm{~s} \mathrm{U}$ is readily taken up and incorporated into newly transcribed RNA $[1,2]$. In mammalian cells, this uptake results in a $0.5 \%$ to $2.3 \%$ 
had no role in study design, data collection and analysis, decision to publish, or preparation of the manuscript.

Competing interests: The authors have declared that no competing interests exist. median $4 \mathrm{sU}$ incorporation rate $[3,4]$ when in the presence of $500 \mu \mathrm{M} 4 \mathrm{sU}$ for $2 \mathrm{hr}$ and $100 \mu \mathrm{M}$ for $24 \mathrm{hr}$. $4 \mathrm{sU}$ differs from uridine by the existence of a sulfur atom rather than an oxygen atom in the $4^{\text {th }}$ carbon position on the nitrogenous base [5]. $4 \mathrm{sU}$ incorporation into RNA assists in the physical or computational isolation of RNA species. The modified nucleotide can be biotinylated to allow for bead capture and subsequent isolation $[1,6]$. The presence of $4 s \mathrm{U}$ in RNA also induces a single nucleotide sequencing artifact that can later be used to identify the presence of $4 \mathrm{sU}$ in an RNA strand $[3,6]$. Despite its widespread use, not much is known about the functional implications of $4 \mathrm{~s} U$ incorporation in downstream RNA processes. Recent studies suggested that labeling at $4 \mathrm{~s} \mathrm{U}$ concentrations $>100 \mu \mathrm{M}$, concentrations commonly used across many different cell types [2], may elicit adverse effects, especially during extended labeling times ( $>12 \mathrm{hrs})$ [7]. Indications of toxicity were also observed at concentrations as low as $50 \mu \mathrm{M}$ [7], presumably because ribosomal RNA synthesis is inhibited, triggering cellular stress response [7]. These observations suggested that $4 \mathrm{~s} \mathrm{U}$ incorporation can negatively impact translation and cellular homeostasis. Given the extensive use of $4 \mathrm{~s} \mathrm{U}$ labeling in the study of nascent pre-mRNA splicing, we set out to determine if intron removal efficiency is influenced by $4 \mathrm{sU}$ incorporation.

Pre-mRNA splicing is an essential biological process that allows for proteomic complexity, despite the relatively small number of protein-coding genes within the human genome [8]. This process is carried out by the spliceosome, a multiprotein/snRNA complex that recognizes and binds to hallmark pre-mRNA sequences. These sequences include both the 5 ' and 3 ' splice sites (ss), the branch point, and the polypyrimidine tract [9]. A single nucleotide change in any of these sequences can alter the efficiency of pre-mRNA recognition by the spliceosome. Thus, it is possible that $4 \mathrm{sU}$ incorporation impacts pre-mRNA splicing.

Using complementary in vitro and cell culture experiments, we show that pre-mRNA splicing can be impacted by the incorporation of $4 \mathrm{~s} U$ into pre-mRNAs. The extent of splicing interference depends on $4 \mathrm{sU}$ incorporation levels and the intrinsic ability of introns to be recognized by the spliceosome. Thus, at high levels of $4 \mathrm{~s} U$ incorporation, weaker exons are more likely to be differentially processed. 4 sU labeling also interfered with in vitro transcription and in vitro pre-mRNA stability at elevated levels of incorporation. We conclude that the presence of $4 s \mathrm{U}$ in nascent RNA can interfere with pre-mRNA splicing. However, the negative effects are only detectable at elevated levels of $4 \mathrm{~s} \mathrm{U}$ incorporation levels and in the context of weak splicing events.

\section{Materials and methods}

\section{In vitro transcription of radiolabeled pre-mRNA}

AdML and $\beta$-Globin, two well-studied DNA constructs that are spliced with ease by the spliceosome when transcribed under normal conditions, were used to generate pre-mRNA for in vitro splicing [10]. Splice site strengths for each of the constructs were determined using MaxEntScanner [11]. After linearizing with BamHI (Promega, R6021) and cleaning/concentrating (Zymo, D4013) AdML and $\beta$-Globin, a $10 \mu \mathrm{L}$ radioactive in vitro transcription reaction was performed using T7 polymerase, $\left[\alpha-{ }^{32} \mathrm{P}\right]$ CTP (Perkin Elmer, BLU008H250UC), and phosphorylated 4-thiouridine (TriLink, N-1025). Different strands of pre-mRNA were generated for each construct that included different quantities of $4 \mathrm{sU}$. The amounts of $4 \mathrm{~s} U$ relative to the total amount of rUTP used in the transcription reaction mixture were $0 \%, 2.5 \%, 30 \%$, and $100 \%$. The resulting pre-mRNA was PAGE purified, eluted, precipitated, and resuspended at $10 \mu \mathrm{L}$ per $100,000 \mathrm{cpm}$ on the Geiger counter. 


\section{In vitro pre-mRNA splicing}

In vitro splicing was carried out by following a well-established protocol [12] in either $12.5 \mu \mathrm{L}$ or $25 \mu \mathrm{L}$ reactions for 0,1 , and 2 hours. Each time point of the he splicing reactions were run out on $6 \%$ polyacrylamide (19:1 acrylamide: bisacrylamide) $7 \mathrm{M}$ urea gels and imaged using a GE Typhoon imager. Splicing efficiency was determined by following the 'percent spliced product' equation in the in vitro splicing protocol [12]. To calculate 'percent spliced product', the band intensities in each lane of the gel that correspond to the unspliced pre-mRNA and the spliced mRNA must be obtained, including correction for background signal. To compute 'percent spliced product' the following equation was used:

Percent Spliced Product $=\left(\frac{\text { Band intensity of spliced } m R N A}{(\text { Band intensity of spliced } m R N A+\text { unspsliced pre }-m R N A)}\right) \times 100$.

Splicing rates were determined by fitting data points to pseudo-first-order decay equations. All resulting gels were analyzed using GelAnalyzer 19.1 software [13], visualized using GraphPad Prism9 software [14] and all statistical analyses were performed with Prism9's built-in statistical analysis tools. The degradation assays were performed identically to the in vitro splicing assays, except we created degradation conditions by omitting the addition of RNasin, CP, and ATP from the reaction mixture and by depleting the nuclear extract of ATP by incubation at room temperature for $30 \mathrm{~min}$ prior to the addition of the labeled RNA. Normalized degradation profiles were calculated by dividing the band intensity of the intact pre-mRNA by the band intensity of the degraded pre-mRNA along the time points of the reaction.

\section{HEK293 metabolic labeling}

HEK293 cells (ATCC, CRL-1573) were grown in 6-well plates at $37^{\circ} \mathrm{C}, 5 \% \mathrm{CO}_{2}$ in a humidified incubator to $80 \%$ confluency for the start of the $2 \mathrm{hr}$ labeling experiments and $40 \%$ confluency for the start of the $24 \mathrm{hr}$ labeling experiments. The cells were grown in DMEM/High Glucose (HyClone, SH30022.01) plus 10\% FBS (GeneClone, 25-550). A metabolic labeling protocol was followed [6] and the experimental HEK293 cells were incubated in the presence of $40 \mu \mathrm{M}$ unphosphorylated 4-thiouridine (Cayman Chemical, CAY16373-5). Total RNA was harvested from the cells using Trizol (Ambion, 15596018) and processed per the metabolic labeling protocol. The resulting pure total RNA was stored in TE at $-80^{\circ} \mathrm{C}$ for further experimentation. Experiments were carried out in biological triplicates.

\section{Endpoint PCR}

Using $1 \mu \mathrm{g}$ of total RNA harvested from the metabolic labeling experiments, cDNAs from three biological triplicates were generated using a first-strand cDNA synthesis kit (Invitrogen, 18080-044) by following the manufacturer-provided protocol. Oligo $d(T)_{16}$ (IDT, 51-01-1506) was used as the primer for the CDNA reaction. PCR primers were designed that flank the exons/regions of interest or using previously described primer sets [15]. Primer sequences are as follows: F_RIOK3_Ex5 CCGGTTCCCACTCCTAAAAAGGGC; R_RIOK3_Ex10 CCAGCATGCCACAGCATGTTATACTCAC; F_TRA2B_Ex1 AGGAAGGTGCAAGAGG TTGG; R_TRA2B_Ex3 TCCGTGAGCACTTCCACTTC; F_CLK2_Ex3 CCGGACATTTAGCCGC TCAT; R_CLK2_Ex6 TGGCCATGGTAGTCAAACCA; F_DDX5_Ex11 ATtGCTACAGATGTGG CCTCC; R_DDX5_Ex12 TGCCTGTTTTGGTACTGCGA. We designed additional sets of primers to investigate both alternative 'Alt' splicing events and constitutive 'Const' splicing events: F_ADAP2_Alt_Ex2 CAGCAGAGTTAAATCTGTGCGAC; R_ADAP2_Alt_Ex4 CTTAGCTCGAATCCATTGTTCC; F_ADAP2_Const_Ex6 AATGCCACCTTCCAGACAGA; 
R_ADAP2_Const_Ex8 TGGCCCAGTCTTTTCCATGA; F_DOLPP1_Alt_Ex5 GCACCAAACAA ACAACGCCA; R_DOLPP1_Alt_Ex6 GGAAGAACTCGGAGACAGGC; F_DOLPP1_Const_Ex2 GTCGGTTTCGTGACCCTCATC; R_DOLPP1_Const_Ex5 CAGGAACCTGGCG TTGTTTG; F_ZNF711_Alt_Ex6 CACCAGTGGACATTCAGTAGC; R_ZNF711_Alt_Ex8 GCTTGACAATCT TCATACCTTCG; F_ZNF711_Const_Ex4 GTGATTCAAGCAGCTGGAGG; R_ZNF711_Const_ Ex6 CATCTTCTCCCGCTGCATTC. PCR was performed after appropriate adjustments in annealing temperature and elongation time were made for each primer set using an Eppendorf Mastercycler Nexus thermal cycler. PCR reactions were run out on $2.0 \%$ agarose gels at $120 \mathrm{~V}$ for 40min and imaged using a Bio-Rad gel doc imager.

\section{Results}

\section{The impact of $4 \mathrm{sU}$ on in vitro splicing efficiencies}

To measure the effect of $4 \mathrm{sU}$ incorporation on pre-mRNA splicing we carried out in vitro and cell culture pre-mRNA splicing analyses. For the in vitro approach, we evaluated the premRNA splicing activities of two well-studied minigene constructs, AdML and $\beta$-Globin, by generating and testing pre-mRNAs with varying amounts of $4 \mathrm{~s} U$ incorporation $(0 \%, 2.5 \%$, $30 \%$, and $100 \% 4 \mathrm{sU}$ incorporation). AdML and $\beta$-Globin minigenes are gold standard in vitro splicing constructs that have been shown to splice efficiently in splicing extract. Both minigenes harbor strong splice sites, with AdML containing a 5' splice site with a MaxEnt score (MES) [11] of 7.9 and a 3' splice site MES of 12.5 , and $\beta$-Globin containing a 5' splice site MES of 8.1 and 3' splice site MES of 9.5 (Fig 1A). Given its higher 3' splice site score, the AdML minigene is considered to be more efficiently spliced than the $\beta$-Globin minigene.

To determine if $4 \mathrm{sU}$ incorporation influences rates of in vitro splicing, splicing efficiencies after $1 \mathrm{hr}$ and $2 \mathrm{hrs}$ were compared between minigene constructs with incorporation rates of $0 \%, 2.5 \%, 30 \%$, and $100 \% 4 \mathrm{sU}$. For $\beta$-Globin we observe a gradual decrease in the average splicing efficiency as the level of $4 \mathrm{sU}$ incorporation in the pre-mRNA increases (Fig $1 \mathrm{~B}$ and $1 \mathrm{C})$. While $4 \mathrm{~s} \mathrm{U}$ incorporation rates of $2.5 \%$ did not change observed splicing rates significantly, higher levels of $4 \mathrm{sU}$ incorporation led to an up to a 3-fold reduction in splicing (Fig 1C). The observations are qualitatively similar for the tested AdML pre-mRNA (Fig 1D). However, considering the range of splicing reduction and variation of the independent experiments, the differences observed did not meet the cutoff for statistical significance.

It is likely that the more striking differences in splicing efficiencies observed for the $\beta$-Globin analysis are the consequence of its intrinsically weaker splice site signals when compared to AdML. We conclude that elevated levels of $4 \mathrm{~s} U$ incorporation into pre-mRNAs can reduce splicing efficiencies and the resulting splicing rates, at least within the context of the timecourse in vitro splicing assays used.

\section{Stability of $4 s U$ containing pre-mRNA}

Modified RNAs are known to be more stable than unmodified RNAs [16, 17]. To evaluate whether the incorporation of $4 \mathrm{sU}$ into RNA alters the stability of the resulting RNA we carried out in vitro degradation assays comparing the fate of fully modified or unmodified RNA samples over a 90 min period. Unmodified AdML RNAs displayed measurable degradation over the 90 min incubation (Fig 2A). Fully modified AdML RNAs did undergo only limited degradation over the same time frame. First-order decay regression of the profiles indicates statistically significant differences between the degradation behaviors (Fig $2 \mathrm{~B}, \mathrm{P}<0.001$ ).

Interestingly, a similar degradation analysis of our other in vitro splicing substrate $\beta$-Globin did not uncover measurable differences between the unmodified $(0 \% 4 \mathrm{sU})$ and the fully $4 \mathrm{sU}$ modified (100\% 4sU) RNAs (data not shown). These results suggest that for the AdML pre- 
A

AdML

$\mathrm{B}$

$\beta$-Globin

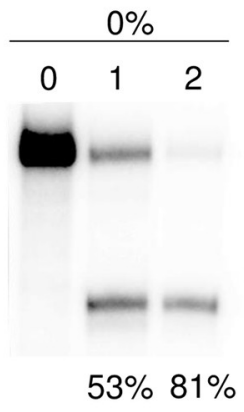

$53 \% 81 \%$

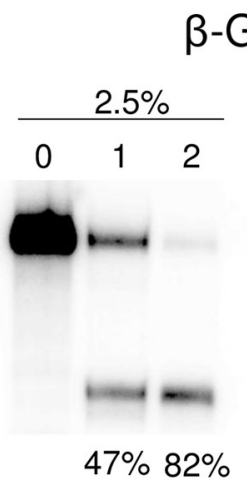

$47 \% 82 \%$

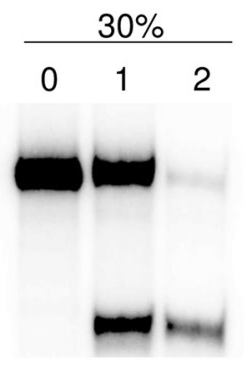

$35 \% 78 \%$

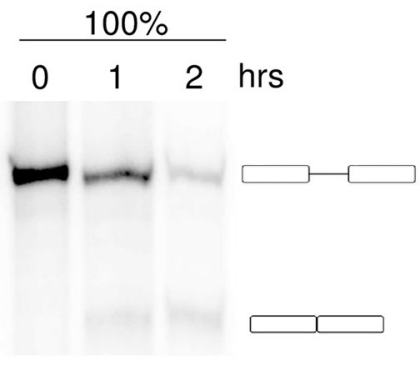

$18 \% 39 \%$
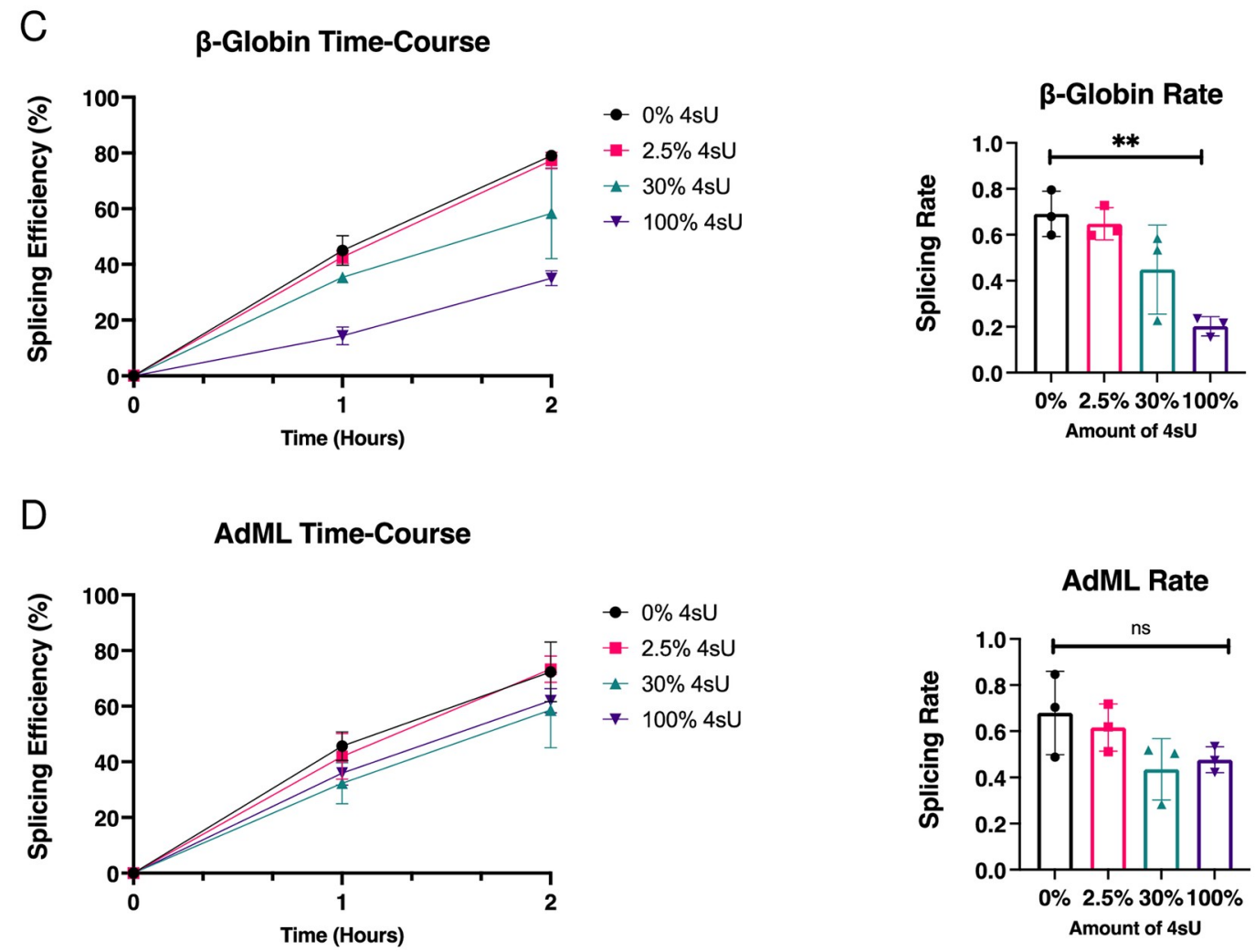

Fig 1. In vitro splicing kinetics of $4 \mathbf{s U}$ labeled RNA. (A) Schematic depictions of the AdML and $\beta$-Globin minigenes used. Exon and intron sizes, splice sites and strengths (MaxEnt score in red) are indicated. (B) Autoradiogram representing time course behavior of $0 \%, 2.5 \%, 30 \%$, and $100 \% 4 \mathrm{sU}$ containing $\beta$-Globin pre-mRNAs. Precursor RNA and spliced products are defined on the right of the gel. The numbers under each lane represents the efficiency of splicing. (C) Graphical representation of $\beta$-Globin time-course splicing assay splicing efficiencies over time $(n=3)$ and the corresponding splicing rate. ${ }^{* * \prime}$ indicates statistically significant difference (ordinary one-way ANOVA, $P=0.0031$ ). (D) Graphical representation of 
AdML time-course splicing assay splicing efficiencies over time $(n=3)$ and the corresponding splicing rate. ' $n$ ' indicates no statistically significant difference (ordinary one-way ANOVA, $\mathrm{P}=0.14$ ).

https://doi.org/10.1371/journal.pone.0257503.g001

mRNA the presence of 4sU promotes RNA stability, similar to other RNA modifications [18]. Just as 2'-O-methyl RNA and phosphorothioate RNA modifications resist nuclease activity [19], 4sU has the potential to limit nuclease attack. The increase in RNA stability (as is the case for AdML, but not for $\beta$-Globin) may introduce a confounding affect when evaluating splicing

A

AdML

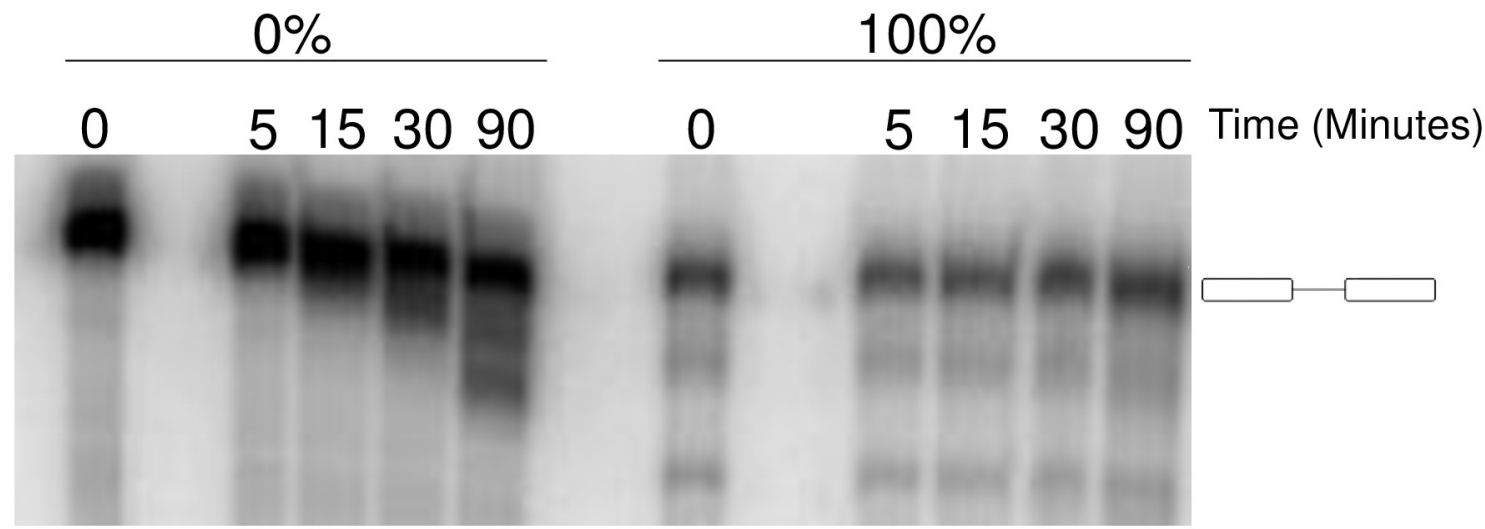

B

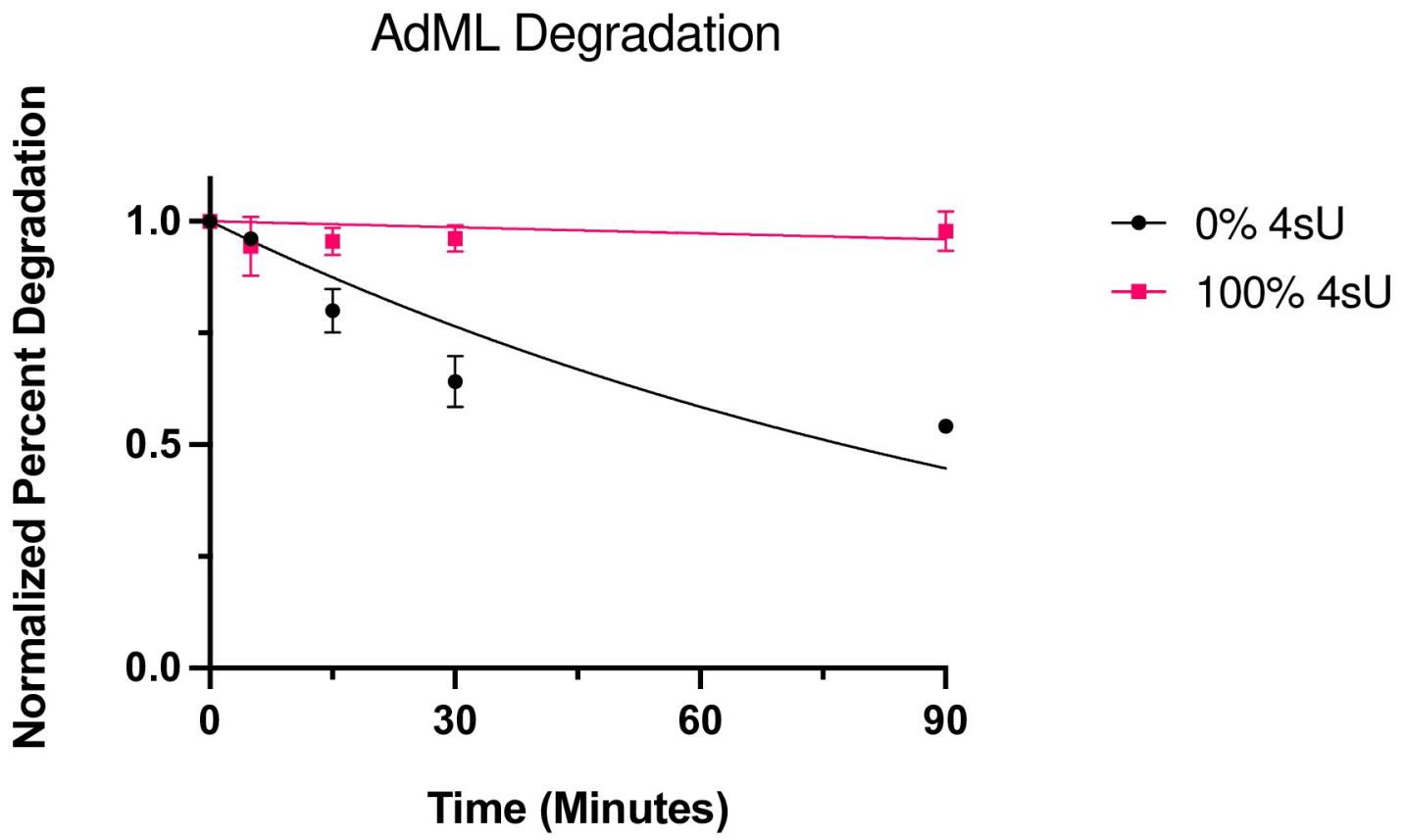

Fig 2. In vitro degradation assay of AdML minigene. (A) Representative autoradiograph of degradation time-course for unmodified and fully modified 4sU AdML pre-mRNA. Time ' 0 ' represents input RNA. Full-length input RNA is defined by the cartoon to the right of the gel. (B) Quantitation of the data shown in (A). First order decay regression of time course data was used for rate determination and statistical analysis.

https://doi.org/10.1371/journal.pone.0257503.g002 
efficiency as described in Fig 1. Thus, the trends observed for AdML splicing at the various $4 \mathrm{~s} U$ incorporation rates may be influenced by altered stability. This is not a concern for the $\beta$ Globin analysis as we did not observe differences in $\beta$-Globin RNA stability.

\section{The effects of $4 \mathrm{~s} U$ on in vitro transcription}

In vitro transcription of AdML and $\beta$-Globin at the defined concentrations of $4 \mathrm{~s} U$ resulted in variable accumulation of abortive transcripts. For example, transcription of AdML led to a marked increase of an abortive transcript with higher incorporation rates of $4 \mathrm{sU}$ (Fig $3 \mathrm{~A}$ ). At $100 \% 4 \mathrm{sU}$ incorporation, the majority of transcripts were abortive, resulting in a truncated RNA.

Qualitatively similar trends were observed when transcribing $\beta$-Globin as multiple abortive transcripts are observed in the $100 \% 4 \mathrm{sU}$ lane (Fig 3B). Apparently, T7 RNA polymerase faced similar, but less extreme difficulties in generating the full $\beta$-Globin pre-mRNA. These observations demonstrate that $4 \mathrm{sU}$ can negatively impact the processivity of in vitro transcription leading to pre-maturely terminated RNA products.

\section{The impact of 4sU on splicing in Hek293 cells}

To investigate the impact of $4 \mathrm{~s} \mathrm{U}$ incorporation on the splicing of endogenous genes we metabolically labeled HEK293 cells. Based on previous reports we used a $4 \mathrm{~s} U$ concentration $(40 \mu \mathrm{M})$ that does not elicit toxic translation effects and we evaluated splicing after $2 \mathrm{hrs}$ and $24 \mathrm{hrs}$ of labeling. These $4 \mathrm{~s} \mathrm{U}$ metabolic labeling conditions are representative of the experimental approaches taken when evaluating nascent pre-mRNA splicing. They are also known to result in a $4 \mathrm{sU}$ incorporation rate of $0.5 \%$ to $2.3 \%[3,4]$. In our analysis, we focused on evaluating the effects of $4 \mathrm{sU}$ on the splicing efficiency of constitutively spliced exons (considered efficient splicing events) and alternatively spliced exons (considered less efficient splicing events).

Our results demonstrate that $4 \mathrm{sU}$ metabolic labeling does not significantly interfere with the splicing of constitutive exons. For all examples tested we observed efficient exon inclusion regardless of the presence or absence of $4 \mathrm{sU}$ in either the $2 \mathrm{hr}$ or $24 \mathrm{hr}$ time points (Fig $4 \mathrm{~A}$ ). These observations suggest that efficient splicing events are not influenced by $4 \mathrm{sU}$ at incorporation rates typically used in cell culture experiments. To determine whether less efficient splicing events are affected by $4 \mathrm{sU}$ labeling we tested a select number of exon skipping events, alternative splice site selection events, and intron retention events. Most of the exon skipping events evaluated did not reveal any significant changes in splicing patterns between the control and $4 \mathrm{sU}$ labeled samples in either the $2 \mathrm{hr}$ or the $24 \mathrm{hr}$ samples (Fig 4B), even though some exon inclusion levels were intrinsically low (Fig 4B, ZNF711). As the only exception we detected a small, but statistically significant decrease in DOLPP1 exon 6 inclusion (Fig 4B). We also detected small, but statistically significant changes in intron retention or alternative 5 ' splice site choice after $24 \mathrm{hrs}$ of $4 \mathrm{~s} \mathrm{U}$ incubation (Fig $5 \mathrm{~A}$ and $5 \mathrm{~B}$ ). At longer exposure to $4 \mathrm{sU}$ labeling, the level of DDX5 intron 11 retention increased from $8 \%$ to $12 \%$. While the magnitude of these changes is small, the increase in intron retention indicates that at the conditions used $4 \mathrm{~s} U$ labeling can have an inhibitory effect on intron excision. Similarly, minor changes in alternative splice site choice are observed for RIOK3 exon 8 after 24hrs of incubation (Fig 5B).

Consistent with our in vitro $4 \mathrm{sU}$ data we conclude that efficiently spliced introns are not significantly affected by the presence of $4 \mathrm{sU}$, at least not at the conditions used. With a few exceptions, the alternative splicing events evaluated did not indicate altered splicing outcomes in the presence of $4 \mathrm{sU}$. Thus, $4 \mathrm{sU}$ labeling at the $40 \mu \mathrm{M}$ conditions used herein does not trigger drastic, widespread, and general changes in alternative splicing. 
A

AdML

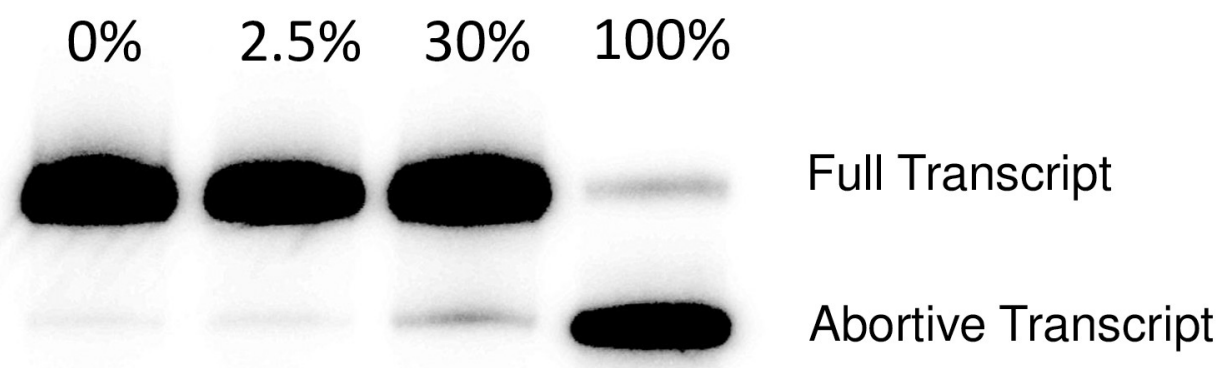

B

$\beta$-Globin

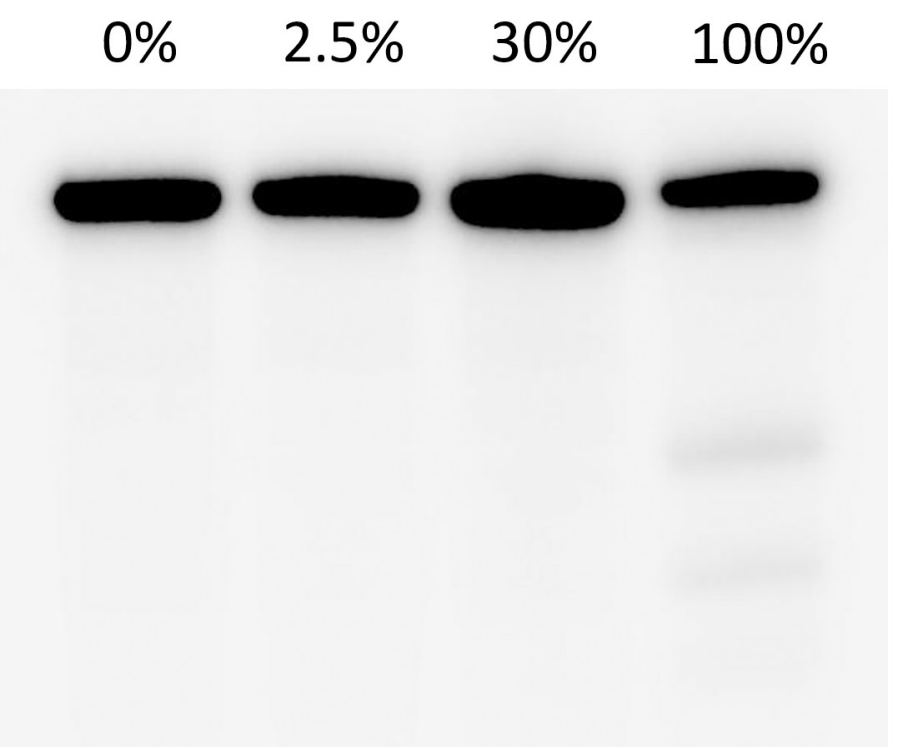

Full Transcript

Abortive Transcript

Abortive Transcript

Fig 3. In vitro transcription of AdML and $\beta$-Globin. Autoradiograph depicting the T7 polymerase transcription profiles of (A) AdML and (B) $\beta$-Globin. Four transcripts of each minigene were created with varying amounts, $0 \%, 2.5 \%, 30 \%$, and $100 \%$, of $4 \mathrm{~s} \mathrm{U}$ incorporated. The full pre-mRNA strands and abortive transcripts in each lane are labeled.

https://doi.org/10.1371/journal.pone.0257503.g003

\section{Discussion}

It is important to investigate whether the tools the scientific community use unintentionally create biases and side effects on the experiments being performed. $4 \mathrm{~s} U$ labeling is a widely used tool in the RNA biology field to capture the processing of nascent RNAs and to evaluate RNA half-lives that is thought to have no consequence on the experimental outcome, aside from the previously reported inhibition of tRNA synthesis and nucleolar stress [7] at elevated concentrations. Here, we show that the incorporation of $4 \mathrm{sU}$ into pre-mRNA strands is not simply inert to the metabolism of an expressed RNA. At elevated levels of $4 \mathrm{sU}$ incorporation 
A

\section{Constitutive Exon}

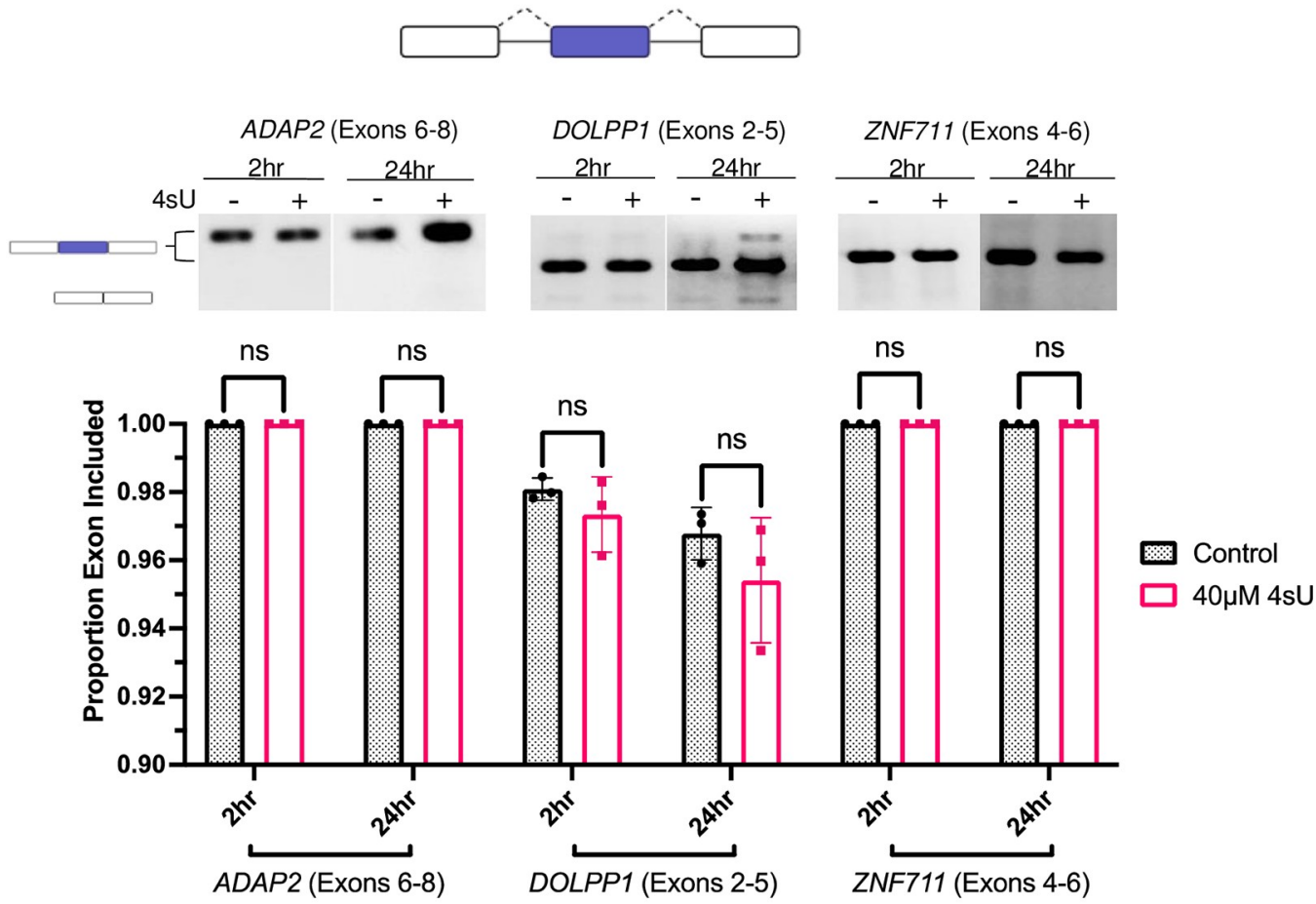

B

\section{Skipped Exon}
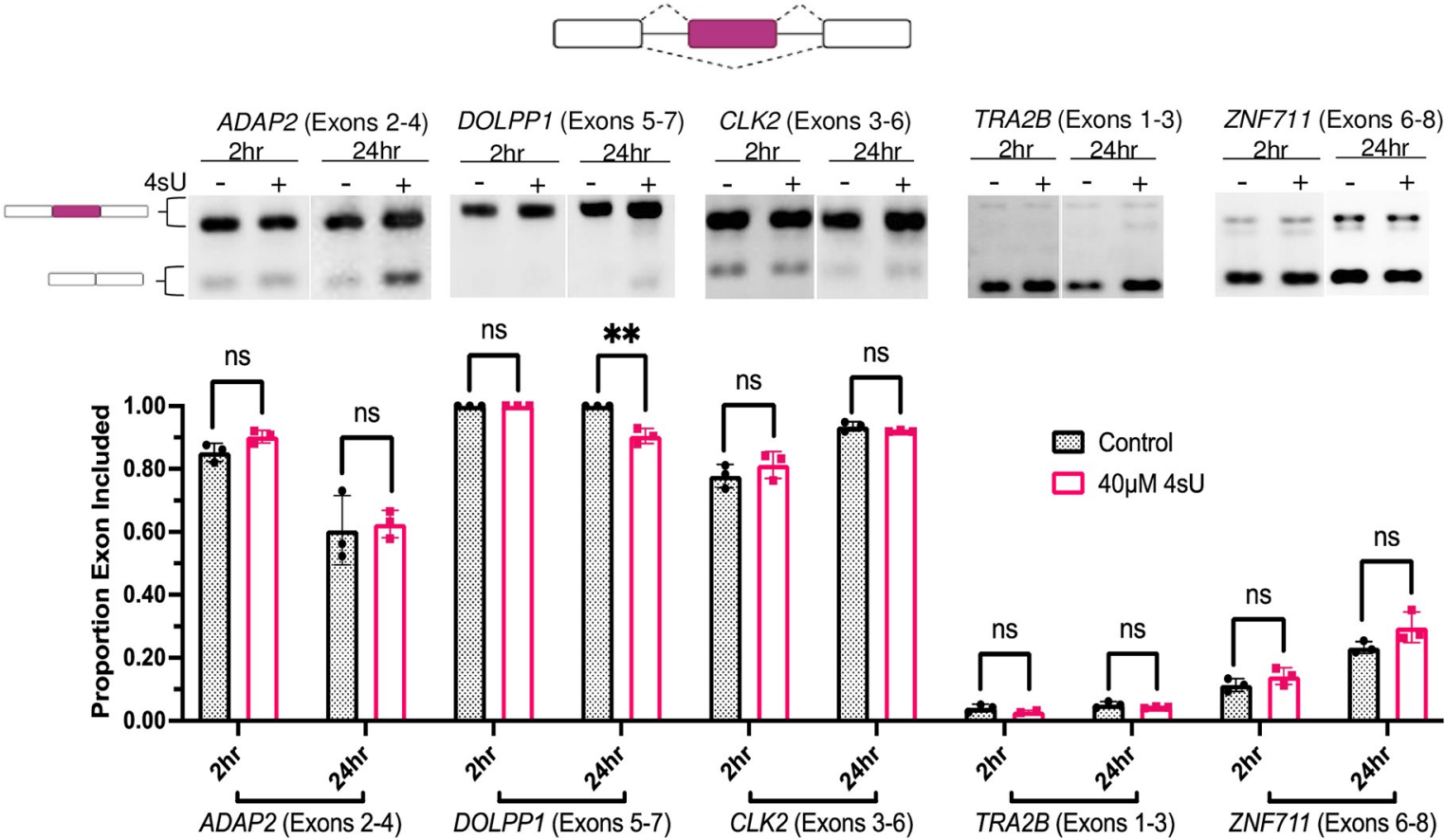

Fig 4. Splicing behavior in cell culture in the presence of $4 \mathbf{s U}$. PCR analysis of (A) constitutive exons within the genes ADAP2, DOLPP1, and ZNF711 and (B) alternatively included exons in genes ADAP2, CLK2, TRA2B, ZNF711, and DOLPP1. Alternative exon inclusion in DOLPP1 (**', $\mathrm{P}=0.0024$ ). ' $n$ s' indicates no statistically significant difference $\mathrm{P}>0.05$ ). The cartoon to the left of each image indicates exon inclusion or exclusion. cDNA from biological triplicates were analyzed after 2 and 24 hours of $4 \mathrm{sU}$ incubation.

https://doi.org/10.1371/journal.pone.0257503.g004 
A
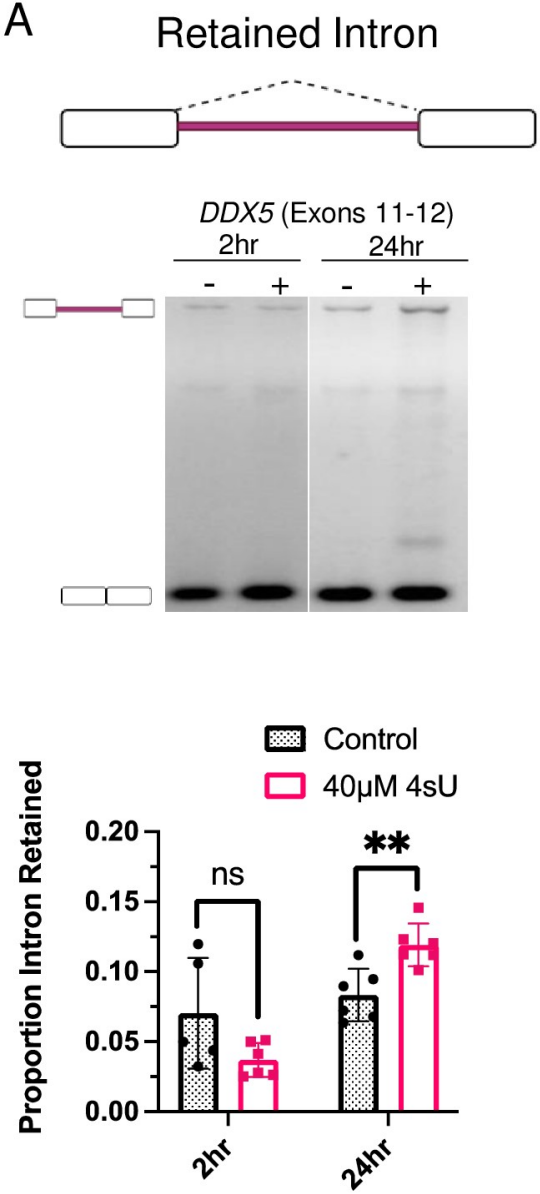

B Alternative 5' Splice Site
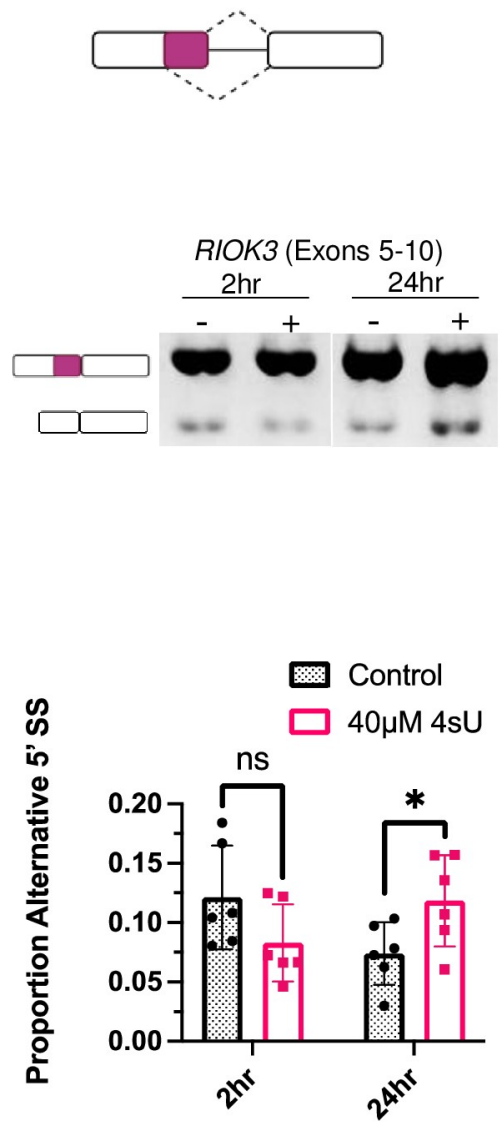

Fig 5. Alternative splicing behavior in cell culture in the presence of $4 \mathbf{s U}$. PCR analysis of $(\mathrm{A})$ intron retention in DDX5 ('ns', $\mathrm{P}=0.08$; '**', $\mathrm{P}=0.0045$ ) and (B) alternative 5'ss selection in RIOK3 ('ns', $\mathrm{P}=0.012$; ${ }^{* *}, \mathrm{P}=0.042$ ). The cartoon to the right of each image defines the alternative splicing products. cDNA samples from biological triplicates were analyzed after 2 and 24 hours of $4 \mathrm{sU}$ incubation.

https://doi.org/10.1371/journal.pone.0257503.g005

RNA transcription, pre-mRNA splicing, and RNA stability can be altered. However, at labeling concentrations typically used in cell culture experiments, the inhibitory effects of $4 \mathrm{sU}$ incorporation on pre-mRNA splicing are minimal, affecting only introns that are already weakly recognized by the spliceosome. Collectively, this data demonstrates that $4 \mathrm{sU}$ is not completely inert when employed as an experimental tool.

Interestingly, at high levels of $4 \mathrm{sU}$ incorporation transcription by $\mathrm{T} 7 \mathrm{RNA}$ polymerase resulted in increased levels of abortive transcripts. This was particularly striking for the AdML minigene (Fig 3A). To identify potential causes for the marked transcription interference we evaluated the RNA sequence of each transcript. The AdML minigene encodes a run of eight consecutive uridines towards the 3' end of the intron. Thus, it is possible that when the polymerase encounters a run of consecutive uridines, the repeated incorporation of $4 \mathrm{~s} U$ could interfere with polymerase processivity, resulting in increased abortive transcripts. The expected size of the AdML abortive transcript is consistent with this possible explanation. The longest stretch of consecutive uridines encoded by the $\beta$-Globin minigene is only six nucleotides long. These observations suggest that longer runs of consecutive uridine induce abortive transcription when the RNA is labeled exclusively with $4 \mathrm{sU}$. The potential implications of this 
transcription interference are straightforward. Incomplete pre-mRNA strands may not be stable, they may not be fully transcribed, and if they are, they could not have proper function.

The results of our experiments demonstrate that $4 \mathrm{sU}$ incorporation can affect pre-mRNA splicing. To what degree do these observations imply that the use of $4 \mathrm{sU}$ in metabolic labeling experiments should be viewed critically? We suggest considering the following aspects when designing metabolic labeling experiments. First, the concentration of $4 \mathrm{~s} U$ used in the labeling approach. Second, the length of metabolic labeling, and third, the splice potential of the exons and introns investigated.

To evaluate the influence of $4 \mathrm{~s} U$ labeling on pre-mRNA splicing we designed experiments that directly measure altered splicing outcomes using in vitro splicing assays. These investigations were complemented with $4 \mathrm{sU}$ labeling approaches of cell cultures, followed by mRNA analysis. Differences in intron excision, exon skipping, or alternative splice site selection rates in cell culture experiments can certainly be triggered by direct splicing effects, however, they can also be representative of altered mRNA stabilities and/or transcription outputs. With these interpretation limitations in mind, our data provide clear guidance when designing and interpreting splicing analyses using $4 \mathrm{~s} \mathrm{U}$ labeling. Increased levels of $4 \mathrm{~s} \mathrm{U}$ incorporation can lead to measurable defects in pre-mRNA splicing, preferentially for weaker splicing events. This is demonstrated in our in vitro splicing assays when pre-mRNAs of different incorporation levels were evaluated. However, our data also demonstrate that for the minigenes used, $4 \mathrm{sU}$ incorporation levels $>30 \%$ need to be achieved to interfere with splicing efficiency. Such elevated levels of $4 \mathrm{sU}$ incorporation cannot be reached when performing cell culture metabolic labeling experiments. The length of $4 \mathrm{sU}$ incubation time is also an important factor when carrying out cell culture metabolic labeling. This is because longer incubation increases the fraction of mRNAs labeled with $4 \mathrm{sU}$, allowing the establishment of steady-state levels of $4 \mathrm{sU}$ mRNAs. An important consideration when analyzing $4 \mathrm{~s} U$ labeling experiments is the identity of the exon or intron evaluated. By definition, constitutive exons are always included in the final mRNA while alternative exons are not. As such, perturbations in recognizing exons, such as the presence of $4 \mathrm{~s} \mathrm{U}$ within the transcript, could have varying influences on alternative and constitutive exon inclusion levels. We observed that constitutive splicing is not affected by the presence of $4 \mathrm{sU}$ at the $40 \mu \mathrm{M}$ concentration used. Most of the alternatively spliced exons evaluated were also not significantly affected by $4 \mathrm{~s} U$ incorporation. Only a limited number of splicing events appeared to indicate altered splice site selection or reduced intron excision. Furthermore, the magnitudes of the observed splice changes were small, suggesting that while statistically significant, biological consequences are expected to be minimal. Our data also suggests that it is possible that the presence of $4 \mathrm{~s} U$ induces the selection of cryptic splice sites. For example, after 24 hrs of $4 \mathrm{sU}$ labeling a novel band appears when evaluating DOLP11 intron retention (Fig 5A). While unconfirmed, this band could represent the use of an alternative splice site. In summary, $4 \mathrm{sU}$ metabolic labeling of cell cultures has the potential to induce altered splicing. While infrequent, at small magnitudes, and only in the context of inefficiently spliced events, appropriate control experiments need to be included to deconvolute splicing differences of tested experimental conditions from those elicited by $4 \mathrm{sU}$ labeling.

It is known that naturally occurring 4-thiouridine modifications increase the stability of some organismal tRNAs [20] and it has been suggested that the incorporation of $4 \mathrm{sU}$ changes the structure of the transcribed RNA. The potential to form secondary structures has been shown to greatly impact exon recognition by the spliceosome [21], so it is entirely possible that $4 \mathrm{~s} \mathrm{U}$ incorporation into RNAs interferes with efficient exon recognition through inducing altered RNA structures.

The results presented here are not damning evidence against the use of $4 \mathrm{sU}$, rather they are akin to a cautionary statement. $4 \mathrm{sU}$ does not broadly impact all aspects of pre-mRNA 
generation and processing, but it does impact those events that are more easily susceptible to change. If $4 \mathrm{~s} U$ is to be used in an experiment, it is important to be mindful of the pre-mRNA splicing events that are being analyzed because a small number of event changes may be caused by the incorporation of $4 \mathrm{~s} \mathrm{U}$ itself.

\section{Supporting information}

S1 Dataset.

(XLSX)

S1 Raw images.

(PDF)

\section{Acknowledgments}

Figures were in part created with Biorender.com.

\section{Author Contributions}

Conceptualization: Klemens J. Hertel.

Formal analysis: Jessie A. C. Altieri.

Funding acquisition: Klemens J. Hertel.

Methodology: Jessie A. C. Altieri.

Resources: Klemens J. Hertel.

Supervision: Klemens J. Hertel.

Validation: Jessie A. C. Altieri.

Writing - original draft: Jessie A. C. Altieri.

Writing - review \& editing: Klemens J. Hertel.

\section{References}

1. Dölken L, Ruzsics Z, Rädle B, Friedel CC, Zimmer R, Mages J, et al. High-resolution gene expression profiling for simultaneous kinetic parameter analysis of RNA synthesis and decay. RNA [Internet]. 2008 [cited 2021 Apr 1]; 14(9):1959-72. Available from: http://www.rnajournal.org/cgi/doi/10.1261/rna. 1136108. PMID: 18658122

2. Duffy EE, Schofield JA, Simon MD. Gaining insight into transcriptome-wide RNA population dynamics through the chemistry of 4-thiouridine [Internet]. Vol. 10, Wiley Interdisciplinary Reviews: RNA. Blackwell Publishing Ltd; 2019 [cited 2021 Mar 29]. p. e1513. /pmc/articles/PMC6768404/ https://doi.org/10. 1002/wrna.1513 PMID: 30370679

3. Herzog VA, Reichholf $B$, Neumann $T$, Rescheneder $P$, Bhat $P$, Burkard TR, et al. Thiol-linked alkylation of RNA to assess expression dynamics. Nat Methods [Internet]. 2017 [cited 2021 Mar 22]; 14 (12):1198-204. Available from: http://www.nature.com/authors/editorial_policies/license.html\#terms https://doi.org/10.1038/nmeth.4435 PMID: 28945705

4. Erhard F, Baptista MAP, Krammer T, Hennig T, Lange M, Arampatzi P, et al. scSLAM-seq reveals core features of transcription dynamics in single cells. Nature [Internet]. 2019 [cited 2021 Apr 14]; 571 (7765):419-23. Available from: https://doi.org/10.1038/s41586-019-1369-y PMID: 31292545

5. National Center for Biotechnology Information. PubChem Compound Summary for CID 5359227. Natl Libr Med [Internet]. 2004 [cited 2021 Apr 1];1-49. Available from: https://pubchem.ncbi.nlm.nih.gov/ compound/4-Thiouridine

6. Garibaldi A, Carranza F, Hertel KJ. Isolation of newly transcribed rna using the metabolic label 4-thiouridine. In: Methods in Molecular Biology [Internet]. Humana Press Inc.; 2017 [cited 2021 Mar 29]. p. 16976. /pmc/articles/PMC5783291/ 
7. Burger K, Mühl B, Kellner M, Rohrmoser M, Gruber-Eber A, Windhager L, et al. 4-Thiouridine inhibits rRNA synthesis and causes a nucleolar stress response. RNA Biol [Internet]. 2013 [cited 2021 Mar 26]; 10(10):1623-30. Available from: https://pubmed.ncbi.nlm.nih.gov/24025460/ https://doi.org/10.4161/ rna.26214

8. Hertel KJ. Combinatorial control of exon recognition [Internet]. Vol. 283, Journal of Biological Chemistry. 2008 [cited 2021 Apr 1]. p. 1211-5. Available from: www.jbc.org https://doi.org/10.1074/jbc. R700035200 PMID: 18024426

9. Moore MJ, Query CC, Sharp PA. Splicing of precursors to messenger RNAs by the spliceosome [Internet]. The RNA world. 1993 [cited 2021 Apr 1]. 303-357 p. www.cshlpress.com/copyright.

10. Green MR, Maniatis T, Melton DA. Human $\beta$-Globin pre-mRNA synthesized in vitro is accurately spliced in xenopus oocyte nuclei. Cell. 1983 Mar 1; 32(3):681-94. https://doi.org/10.1016/0092-8674(83) 90054-5 PMID: 6550524

11. Yeo G, Burge CB. Maximum entropy modeling of short sequence motifs with applications to RNA splicing signals. In: Journal of Computational Biology [Internet]. 2004 [cited 2021 Mar 23]. p. 377-94. Available from: www.liebertpub.com https://doi.org/10.1089/1066527041410418 PMID: 15285897

12. Movassat $M$, Mueller WF, Hertel $\mathrm{KJ}$. In vitro assay of pre-mRNA splicing in mammalian nuclear extract. Methods Mol Biol [Internet]. 2014 [cited 2021 Mar 19]; 1126:151-60. Available from: https://link. springer.com/protocol/10.1007/978-1-62703-980-2_11 PMID: 24549662

13. Lazar IJ, Lazar IS. GelAnalyzer 19.1 [Internet]. [cited 2021 Aug 11]. http://www.gelanalyzer.com

14. GraphPad. Prism [Internet]. [cited 2021 Aug 11]. https://www.graphpad.com/scientific-software/prism/

15. Havranek KE, White LA, Lanchy J-M, Lodmell JS. Transcriptome profiling in Rift Valley fever virus infected cells reveals modified transcriptional and alternative splicing programs. Ikegami T, editor. PLoS One [Internet]. 2019 May 28 [cited 2021 Mar 22]; 14(5):e0217497. Available from: https://dx.plos. org/10.1371/journal.pone.0217497 PMID: 31136639

16. Meng M, Schmidtgall B, Ducho $\mathrm{C}$. Enhanced stability of DNA oligonucleotides with partially zwitterionic backbone structures in biological media. Molecules [Internet]. 2018 Nov 10 [cited 2021 Jul 8]; 23(11). Available from: /pmc/articles/PMC6278555/ https://doi.org/10.3390/molecules23112941 PMID: 30423832

17. Dimitrova DG, Teysset $\mathrm{L}$, Carré C. RNA 2'-O-Methylation $(\mathrm{Nm})$ modification in human diseases. Genes (Basel) [Internet]. 2019 Feb 1 [cited 2021 Jul 8]; 10(2). Available from:/pmc/articles/PMC6409641/ https://doi.org/10.3390/genes10020117 PMID: 30764532

18. Dagle JM, Weeks DL, Walder JA. Pathways of Degradation and Mechanism of Action of Antisense Oligonucleotides in Xenopus laevis Embryos. Antisense Res Dev [Internet]. 1991 Apr 2 [cited 2021 Jul 8]; 1(1):11-20. Available from: https://www.liebertpub.com/doi/abs/10.1089/ard.1991.1.11 PMID: 1668307

19. IDT. Modifications that block nuclease degradation [Internet]. 2016 [cited 2021 Jul 8]. https://www. idtdna.com/pages/education/decoded/article/modification-highlight-modifications-that-block-nucleasedegradation

20. Čavužić M, Liu Y. Biosynthesis of sulfur-containing tRNA modifications: A comparison of bacterial, archaeal, and eukaryotic pathways [Internet]. Vol. 7, Biomolecules. MDPI AG; 2017 [cited 2021 Mar 26]. /pmc/articles/PMC5372739/

21. Hiller M, Zhang Z, Backofen R, Stamm S. Pre-mRNA secondary structures influence exon recognition. Hayashizaki Y, editor. PLoS Genet [Internet]. 2007 Nov 16 [cited 2021 Mar 26]; 3(11):2147-55. Available from: https://dx.plos.org/10.1371/journal.pgen.0030204. PMID: 18020710 